\title{
Analysis of Noncontact Squeegeeing Device for Liquid Toner Electrophotography*
}

\author{
Shuzo HIRAHARA ${ }^{* *}$, Norio YOSHIKAWA ${ }^{* * *}$, Hideki NUKADA****, \\ Haruhi OH-OKA ${ }^{* * * *}$ and Masahiro HOSOYA ${ }^{* * * *}$
}

\begin{abstract}
A noncontact squeegeeing device is used just after the development process in a liquid toner electrophotographic printing system. By using capillary forces, this device removes almost all of the surplus liquid adhering to the photoreceptor surface. Though the device has a simple structure and has even been used in actual systems, there is not much information regarding the mechanism of the device available in the literature, and the mechanism does not seem to have been theoretically analyzed. In this paper, some equations are derived from the lubrication approximation theory based on the condition that total liquid flow is zero. The calculation results obtained using these equations show good agreement with the experimental results for the variation of the characteristics of the squeegeeing efficiency with the circumferential surface velocities of rollers. The proposed equations make the calculation of the squeegeeing characteristics easy and thus facilitate improvement of the device and further development of the system.
\end{abstract}

Key Words: Fluid Dynamics, Viscous Flow, Pressure Distribution, Squeegee, Lubrication Approximation, Liquid Toner Electrophotography

\section{Introduction}

An improved electrophotographic printer system that uses a liquid toner (wet process) has been developed ${ }^{(1)-(5)}$. The dry process is the most commonly used electrophotographic process today and employs powder toner. In the dry process, toner images developed on a photoreceptor are transferred to an intermediate medium or to the printing medium (paper) directly. On the contrary, in the newly developed wet process, two additional devices are needed between the developing and transfer processes to remove the surplus liquid, which serves as the carrier fluid (dispersion medium) for the toner particles in the developer. As shown in Fig. 1, the first is the "squeegeeing device", which removes the surplus liquid mechanically. Immedi-

* Received 15th July, 2003 (No. 03-4097)

** Graduate School of Science and Technology, Keio University, 3-14-1 Hiyoshi, Kohoku-ku, Yokohama, Kanagawa 223-8522, Japan. E-mail: hirahara@mnt.appi.keio.ac.jp

*** Toshiba Corporation Digital Media Network Company, 29 Suehiro-cho, Ome, Tokyo 198-8710, Japan

**** Corporate Research \& Development Center, Toshiba Corporation, 1 Komukai Toshiba-cho, Saiwai-ku, Kawasaki, Kanagawa 212-8582, Japan ately after squeegeeing, however, the removal of liquid is insufficient because the toner particles are accompanied by liquid corresponding to more than $50 \mathrm{wt} \%$ in capillary tubes or crevices. To get good quality transferred images, the toner images must be in the half dried condition, with a liquid content of 5 to $15 \mathrm{wt} \%$, before arriving at the transfer process ${ }^{(2),(4),(5)}$. Therefore a "drying device" is needed in the second device, where the residual

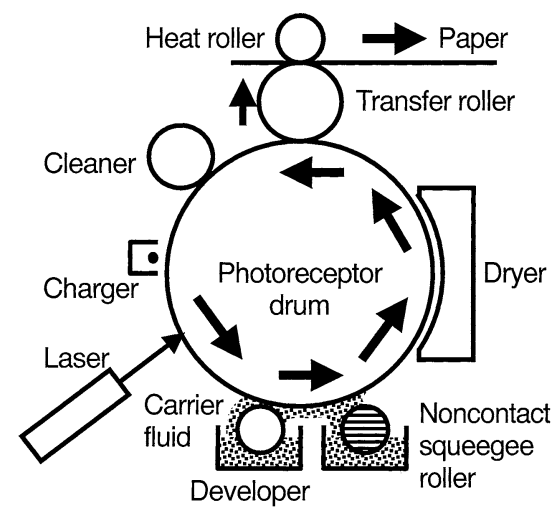

Fig. 1 Liquid toner electrophotography using a noncontact squeegeeing process 


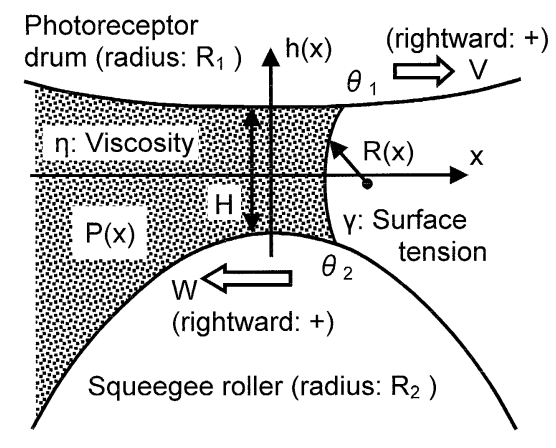

Fig. 2 Liquid surface shape in the gap between rollers and the parameters used in the analysis

liquid is reduced to the optimum level by forced evaporation. A schematic cross-section of the structure of the squeegeeing area is shown in Fig. 2. A gap of several tens of $\mu \mathrm{m}$ to $100 \mu \mathrm{m}$ is present between the photoreceptor drum and the squeegee roller. When the system starts and the rollers rotate, the liquid enters the gap as a result of the capillary force and forms a meniscus (air-liquid interface) at the front end. When the soaked photoreceptor surface passes through this gap, the surplus liquid is sucked into meniscus.

In spite of its simple construction, the noncontact squeegeeing device has the advantage that the toner image is not subject to mechanical damage, such as scratch noise ${ }^{(6)}$. In the field of fluid lubrication, some studies have reported a cavitation problem related to the rupture of the lubrication oil film, leading to burnout of the bearings ${ }^{(7),(8)}$. In these studies, the liquid flow in the gap between two rollers has been analyzed and discussed. However, there are no descriptions for the condition where the liquid flow is completely stopped. Therefore theoretical analysis is required for the design and improvement of this squeegeeing device. The mechanism and the principle of the noncontact squeegeeing process are analyzed in this paper to identify the factors that affect the characteristics the most and to determine whether the effectiveness of the method is limited to a certain range.

\section{Equations Describing the Squeegeeing Character- istics}

Generally, the Navier-Stokes equation is used as the dynamic equation for viscous fluids.

$$
\left\{\begin{aligned}
& \rho \cdot \frac{\partial u}{\partial t}==-\frac{\partial p}{\partial x}+\eta\left(\frac{\partial^{2} u}{\partial x^{2}}+\frac{\partial^{2} u}{\partial y^{2}}+\frac{\partial^{2} u}{\partial z^{2}}\right) \\
& \rho \cdot \frac{\partial v}{\partial t}=-\frac{\partial p}{\partial y}+\eta\left(\frac{\partial^{2} v}{\partial x^{2}}+\frac{\partial^{2} v}{\partial y^{2}}+\frac{\partial^{2} v}{\partial z^{2}}\right) \\
& \rho \cdot \frac{\partial w}{\partial t}=-\frac{\partial p}{\partial z}+\eta\left(\frac{\partial^{2} w}{\partial x^{2}}+\frac{\partial^{2} w}{\partial y^{2}}+\frac{\partial^{2} w}{\partial z^{2}}\right) \\
& \rho: \text { Density of the fluid } \\
& p: \text { Pressure }
\end{aligned}\right.
$$

\author{
$\eta$ : Coefficient of viscosity of the fluid \\ $u, v, w$ : Fluid flow velocities in the $x, y$, and $z$ \\ directions, respectively
}

The lubrication approximation theory presented by Reynolds $^{(9)}$ can be applied in this analysis because the gap between the two rollers, as can be seen in Fig. 2, is sufficiently thin compared with the length in the flow direction. There is no need to consider the EHL (Elastohydrodynamic lubrication) theory ${ }^{(10)}$ because the thickness of the flow is obviously greater than 10 times the roller surface roughness.

Reynolds' equation is obtained from the lubrication approximation theory as follows.

$$
\frac{1}{\eta} \cdot \frac{d P}{d x}=\frac{h^{2}}{12}\left(\frac{V+W}{2}\right)-12 \frac{q}{h^{3}}
$$

$x$ : Distance from the minimum gap point

$V$ : Circumferential surface velocity of the upper roller (photoreceptor drum)

$W$ : Circumferential surface velocity of the lower roller (squeegee roller)

$P(x)$ : Pressure

$q$ : Total flux across any cross section of the liquid flow

$h(x)$ : Distance between the roller surfaces measured parallel to the minimum gap line

It is assumed that the liquid continuously fills the flow path and that the pressure at minus infinity in the $x$ direction (left end in Fig. 2) is zero for the condition that the atmospheric pressure is subtracted as the bias component. Equation (3) below is obtained when Eq. (2) is integrated and solved for the condition that the total flux $(q)$ is zero when squeegeeing works effectively.

$$
P(x)=3 \eta(V+W) \frac{A^{2}}{H^{2}}\left\{\frac{x}{x^{2}+A^{2}}+\frac{1}{A}\left(\frac{\pi}{2}+\arctan \frac{x}{A}\right)\right\}
$$

$$
\begin{aligned}
& A:=\sqrt{2 H \frac{R_{1} R_{2}}{R_{1}+R_{2}}} \\
& H: \text { Minimum gap length }(=h(0)) \\
& R_{1}: \text { Photoreceptor drum radius } \\
& R_{2}: \text { Squeegee roller radius }
\end{aligned}
$$

Equation (3) holds when all the space is filled with fluid. But in the noncontact squeegeeing state, half the space is filled with air and a meniscus is formed in the gap between the rollers. In the no-flux condition assumed above, the inner flux pressure must be balanced by Laplace liquid surface pressure expressed as the liquid surface tension divided by the radius of curvature of the meniscus surface. The dynamic contact angle is assumed to be al- 
most the same as the static contact angle, and the crosssectional figure of the meniscus surface is assumed to be the arc of a circle with a radius $R$. The radius of curvature of the meniscus surface can be express as follows.

$$
R(x)=\frac{H}{A^{2}} \cdot \frac{x^{2}+A^{2}}{\cos \theta_{1}+\cos \theta_{2}}
$$

$R(x)$ : Radius of curvature of the meniscus surface

$\theta_{1}$ : Contact angle of the photoreceptor drum surface

$\theta_{2}$ : Contact angle of the squeegee roller surface

The pressure balance is expressed by Eq. (5) below.

$$
P(x)+\frac{\gamma}{R(x)}=0
$$

$\gamma:$ Surface tension of the liquid

Substitution of Eq. (3) and Eq. (4) into Eq. (5) yields the following equation.

$$
\begin{gathered}
3 \eta \frac{(V+W)}{H}\left[\frac{x}{A}+\left\{\left(\frac{x}{A}\right)^{2}+1\right\}\left(\frac{\pi}{2}+\arctan \frac{x}{A}\right)\right] \\
+\frac{\gamma}{A}\left(\cos \theta_{1}+\cos \theta_{2}\right)=0
\end{gathered}
$$

Equation (6) expresses the relationship between the meniscus position $x$ and the sum of velocities $(V+W)$. Using the values in Table 1 and the derived equations, $P(x), \gamma / R(x)$, and $P(x)+\gamma / R(x)$ are calculated and plotted in Fig. 3. In this graph, it is shown that the meniscus is formed at a position $159 \mu \mathrm{m}$ outside (toward the right in

Table 1 Constants, variables, and parameters used in the calculations

\begin{tabular}{|l|c|l|l|}
\hline \multicolumn{1}{|c|}{ Item } & Symbol & \multicolumn{1}{|c|}{ Value } & \multicolumn{1}{|c|}{ Unit } \\
\hline Surface tension of liquid (Oil-based) & $\mathrm{Y}$ & 0.0259 & $\mathrm{~N} / \mathrm{m}$ \\
\hline Viscosity of liquid (Oil-based) & $\eta$ & 0.00161 & $\mathrm{~Pa} \cdot \mathrm{s}$ \\
\hline Radius of photoreceptor drum & $\mathrm{R}_{1}$ & 0.075 & $\mathrm{~m}$ \\
\hline Velocity of photoreceptor drum surface & $\mathrm{V}$ & (variable) & $\mathrm{m} / \mathrm{s}$ \\
\hline Contact angle of liquid to photoreceptor & $\theta_{1}$ & 13 & degrees \\
\hline Radius of squeegee roller & $\mathrm{R}_{2}$ & 0.0085 & $\mathrm{~m}$ \\
\hline Velocity of squeegee roller surface & $\mathrm{W}$ & (variable) & $\mathrm{m} / \mathrm{s}$ \\
\hline Contact angle of liquid to squeegee roller & $\theta_{2}$ & 5 & degrees \\
\hline Gap between rollers & $\mathrm{H}$ & 0.000030 & $\mathrm{~m}$ \\
\hline
\end{tabular}

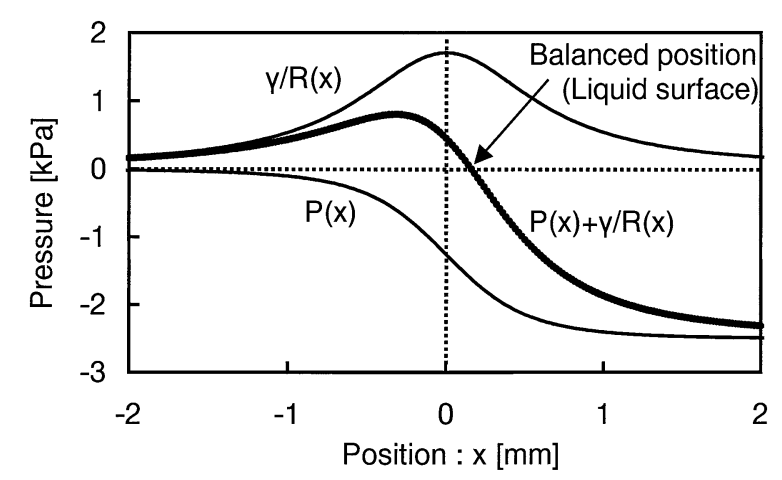

Fig. 3 Pressure balance equation $(P+\gamma / R=0)$ used to derive the liquid surface position
Fig. 2) the minimum gap point. Though $\theta_{1}$ and $\theta_{2}$ are used as the constants in this section and the next section, but these will be discussed about the treatment as the variables affected with the roller speed in the latter part of section 5 .

\section{Applicable Range}

To obtain a solution for Eq. (5), $P(x)$ must be negative, because $\gamma / R(x)$ is always positive. This leads to the next condition.

$$
(V+W)<0
$$

For condition (7) to hold, the following two conditions must be satisfied at the same time. The first is that the movement direction of the surface of the squeegee roller is opposite to that of the photoreceptor drum. And the second is that the movement speed of the surface of the squeegee roller $(W)$ is higher than that of the photoreceptor drum $(V)$. This condition can be expressed as $|V|<|W|$ or $|V / W|<1$. Figure 4 shows the graphs of pressure balance Eq. (6) calculated for the parameter $(V+W)$. For the other constants, the values given in Table 1 are used. It is observed that the pressure balance equation, $P(x)+\gamma / R(x)=0$, has a solution only if $(V+W)$ is restricted to a negative value. Therefore, condition (7) gives the maximum limit of $(V+W)$.

If $|W|$ increases ( $W$ actually decreases, because it is negative) within the range where condition (7) is satisfied, the pressure balance position $x$ will move toward the liquid supply side (left side in Figs. 2, 3, and 4), the negative pressure $P(x)$ in Eq. (3) will fall to a lower value (the absolute value will increase), and liquid suction force will become stronger. It is considered that the stronger the suction, the greater the effectivity of squeegeeing.

The pressure balance is thus maintained by an antagonistic system composed of the first term $P(x)$ and the second term $\gamma / R(x)$ in Eq. (5). If the meniscus position $x$ moves, the antagonistic system remains stable as long as $x$ is in the positive range $(0<x)$. If, however, $x$ goes to 0 , the second term $\gamma / R(x)$ becomes quasi-stable, with $x$ becoming extremely sensitive to pressure fluctuations,

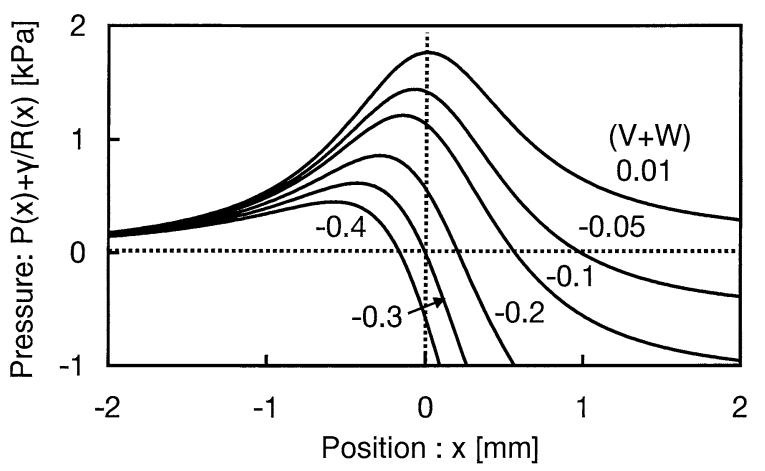

Fig. 4 Variation of the pressure balance with the parameter $V+$ W 


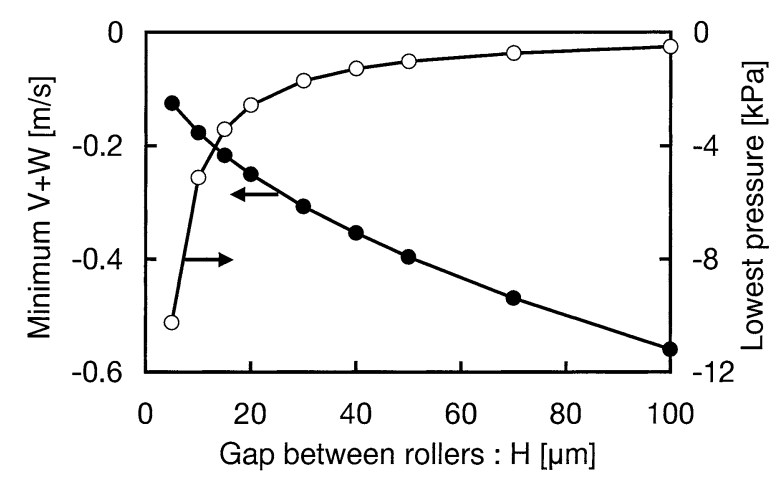

Fig. 5 Calculated variation of the minimum $V+W$ and lowest negative pressure with the gap between the rollers

and the movement in the $x$ direction becoming difficult to determine. $R(x)$ becomes unstable when $x$ becomes 0 or negative. Therefore, for the liquid to be squeegeed effectively, $x$ must be in the positive range, toward the right of the minimum gap point in Figs. 2, 3, and 4. For the airliquid interface to be static and stable, Eq. (8) below, derived by M.D. Savage ${ }^{(9)}$, must be satisfied. The inequality $0 \leqq x$ is a mathematically sufficient condition for Eq. (8) to be satisfied.

$$
\frac{d}{d x}\left\{P(x)+\frac{\gamma}{R(x)}\right\}<0
$$

Substitution of 0 for $x$ in Eq. (6) yields the next inequality.

$$
-\frac{2}{3 \pi} \cdot \frac{\gamma}{\eta} \cdot \frac{H}{A}\left(\cos \theta_{1}+\cos \theta_{2}\right)<(V+W)
$$

This gives the minimum limit value of $(V+W)$, which is calculated to be $-0.307 \mathrm{~m} / \mathrm{s}$ in the model case of Table 1 .

Equation (9) indicates that the minimum value of $(V+$ $W$ ) is a function of $R_{1}$ (photoreceptor drum radius), $R_{2}$ (squeegee roller radius), and $H$ (minimum gap distance). In Fig. 5, the minimum value of $(V+W)$ is plotted as a function of $H$, with $R_{1}$ and $R_{2}$ treated as constants with the values given in Table 1 . In the same figure, the maximum suction force $(P(0)$, negative pressure), which represents the squeegeeing capability when $(V+W)$ is minimum, is also plotted as a function of $H$. The maximum limit for $(V+W)$ given by Eq. (7) and the minimum limit given by Eq. (9) for $(V+W)$ can be summarized as follows.

$$
-\frac{2}{3 \pi} \cdot \frac{\gamma}{\eta} \cdot \frac{H}{A}\left(\cos \theta_{1}+\cos \theta_{2}\right)<(V+W)<0
$$

$$
\text { Here, } W<0<V \text { and }|V|<|W| \text {. }
$$

Thus a conditional inequality for the existence of a static and stable meniscus is finally derived as a function of the variable $(V+W)$ alone.

\section{Comparison with Experimental Data and Discus- sion}

A test machine with the specifications shown in Table 1 was used to perform experiments to measure the thickness of the residual liquid on the photoreceptor drum

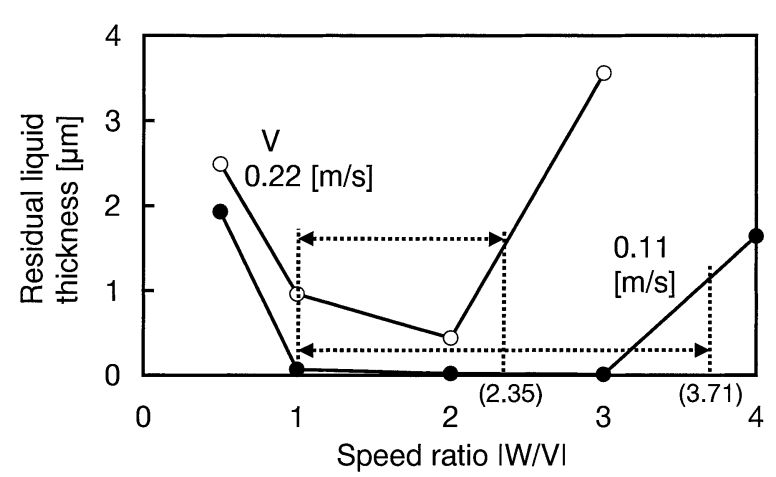

Fig. 6 Experimentally observed variation of the residual liquid thickness (after the squeegeeing process) with the speed ratio $|W / V|$. The dotted lines indicate the theoretical limits of the range within which squeegeeing is effective.

after the squeegeeing device. The liquid thickness was measured by first weighing the liquid scraped from the photoreceptor surface using a rubber blade, then dividing the weight by the liquid density to obtain the volume, and finally dividing the volume by the surface area to calculate the thickness. Figure 6 shows the variation of the measured thickness of the residual liquid with $|W / V|$, which is the ratio of the surface velocities of two rollers. The characteristics are plotted for $V=0.11 \mathrm{~m} / \mathrm{s}$ and $V=0.22 \mathrm{~m} / \mathrm{s}$ using closed circles and open circles, respectively.

Using the values in Table 1, the inequalities (10) are transformed into the following practical expression.

$$
1<\left|\frac{W}{V}\right|<1+\frac{0.298}{V}
$$

The limits calculated by the inequalities (11) for $V=$ $0.11 \mathrm{~m} / \mathrm{s}$ and $V=0.22 \mathrm{~m} / \mathrm{s}$ are illustrated with three vertical dotted lines in Fig. 6. Because the lower limit is always 1 in the inequalities (11), the lower boundary line crosses the horizontal axis at $|W / V|=1$ for both values of $V$. The upper boundary line crosses the horizontal axis at $|W / V|=2.35$ for $V=0.22 \mathrm{~m} / \mathrm{s}$ and at $|W / V|=3.71$ for $V=0.11 \mathrm{~m} / \mathrm{s}$. It can clearly be seen that both the characteristic curves take minimum values within the dotted vertical lines. Therefore, the property derived from theoretical analysis is verified by the experimental data.

Figure 6 also reveals another important feature. The minimum thickness is as small as $10 \mathrm{~nm}$ for $V=0.11 \mathrm{~m} / \mathrm{s}$, indicating that the squeegeeing is extremely effective. For $V=0.22 \mathrm{~m} / \mathrm{s}$, however, the minimum residual liquid film thickness is greater than $440 \mathrm{~nm}$, indicating that the squeegeeing is not as effective. This phenomenon is discussed below.

Many studies have been performed on the dynamic contact angle caused by the surface roughness or the orientation of surface molecules ${ }^{(11),(12)}$. Generally, there is a difference of about $10^{\circ}$ in the contact angles for advancing and receding. The difference can become as high as $100^{\circ}$ due to non-uniform contents or heterogeneous ma- 


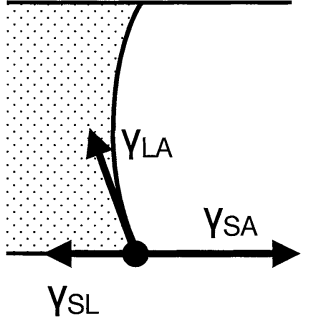

(a)

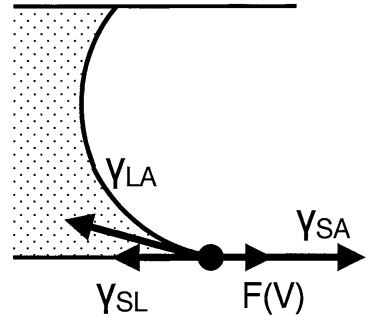

(b)
Fig. 7 (a) Three forces are in a balanced state in accordance with Young's equation, when the roller speed $V$ is assumed to be negligible. (b) When the roller speed $V$ increases, the balancing relation has to be modified by incorporating the fourth factor $F(V)$.

terials $^{(13)}$. The contact angle formed at the boundary of two kinds of liquids in a solid tube has also been reported to have speed dependence ${ }^{(14)}$. Another study has demonstrated the speed dependence of the advancing contact angle formed at the air-liquid interface in a glass capillary tube ${ }^{(15)}$. But there seem to be no studies on receding movement. When a static air-liquid interface exists at a solid surface, as shown in Fig. 7 (a), the contact angle is decided by the balance of three surface forces as expressed by Young's equation as follows.

$$
\begin{aligned}
& \gamma_{S L}+\gamma_{L A} \cdot \cos \theta=\gamma_{S A} \\
& \gamma_{S L}: \text { Solid-liquid interface energy } \\
& \gamma_{L A}: \text { Air-liquid interface energy } \\
&(=\gamma: \text { surface tension }) \\
& \gamma_{S A}: \text { Air-solid interface energy } \\
&(: \text { surface energy }) \\
& \theta: \text { Static contact angle }
\end{aligned}
$$

It was reported ${ }^{(16)}$ that theadvancing contact angles lie on the same asymptotic curve to $(\eta V / \gamma)^{1 / 3}$ for many materials measured over the wide speed range. Therefore, the characteristic for advancing contact angle is represented as the next equation.

$$
\phi=\theta+\left(\frac{\eta V}{\gamma}\right)^{\frac{1}{3}}
$$

Assuming that the characteristic curve can be extrapolated over the static angle $\theta$ the curve in the range of receding contact angle is expressed as the next.

$$
\phi=\theta-\left(\frac{\eta V}{\gamma}\right)^{\frac{1}{3}}
$$

Equation (14) indicates that the receding contact angle $\phi=\theta-(\eta V / \gamma)^{1 / 3}$ decreases gradually as the velocity $V$ increases, and it may eventually reach zero $(\cos \phi=1)$. If $\phi$ reaches zero, the squeegeeing system cannot maintain the state of balance, and allows a thick liquid film to pass through. The experimental characteristic curve

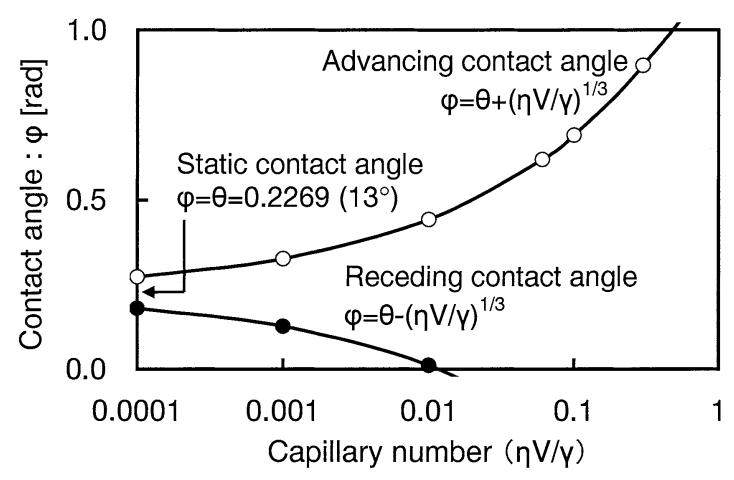

Fig. 8 Calculated dynamic contact angle

for $V=0.22 \mathrm{~m} / \mathrm{s}$ in Fig. 6 can be explained by this phenomenon. This means that, to achieve effective squeegeeing, the surface speed of photoreceptor $V$ must not exceed the following maximum limit.

$$
V<\frac{\gamma}{\eta} \theta^{3}
$$

It is shown in Fig. 8 that dynamic contact angles are calculated using Eqs. (13) and (14) with the liquid surface tension $\gamma$, viscosity $\eta$, and contact angle $\theta$ listed in Table 1 . The maximum limit of the surface speed of photoreceptor $V$ was calculated to be $0.188 \mathrm{~m} / \mathrm{s}$, at which the characteristic curve $\phi=\theta-(\eta V / \gamma)^{1 / 3}$ intercepts the horizontal axis in Fig. 8. This calculated speed of $0.188 \mathrm{~m} / \mathrm{s}$ lies between $0.11 \mathrm{~m} / \mathrm{s}$, where squeegeeing was found to be sufficient, and $0.22 \mathrm{~m} / \mathrm{s}$, where squeegeeing was found to be insufficient. Therefore, it can be concluded that the difference of the squeegeeing effect between these speeds is a result of the reduction of the receding contact angle, which depends on the surface speed of the photoreceptor.

\section{Conclusion}

The mechanism and characteristics of the noncontact squeegeeing process used in liquid toner electrophotography are clarified by theoretical analysis. Equation (6), which describes the property of the squeegeeing device, was derived from the balance of the hydrodynamic negative pressure with the surface tension pressure causing at the curved air-liquid interface. Following knowledge has been obtained by comparison with the experimental results.

(1) The squeegeeing effect completely works in the range of the optimum speed condition which is described in Eq. (10) for the sum of the photoreceptor drum speed $V$ and the squeegee roller speed $W$.

(2) It is supposed that the upper limit of process speed depends on the dynamic (receding) contact angle characteristics of the liquid to photoreceptor as expressed in Eq. (14) which is the function of Capillary number $\eta V / \gamma$.

These theoretical equations are expected to facilitate the improvement and further development of the design of 
noncontact squeegeeing devices.

\section{Acknowledgements}

We are grateful to Dr. Haruyuki Minamitani, Professor of Graduate School of Science and Technology at Keio University, for his invaluable advice from the viewpoint of microfluidics.

\section{References}

( 1 ) Yagi, H., Shinjo, Y., Oh-oka, H., Saito, M., Ishii, K., Takasu, I. and Hosoya, M., Image-on-Image Color Process Using Liquid Toner, Proceedings of the 2000 International Conference on Digital Printing Technologies (:NIP16) (IS\&T), (2000), pp.246-250.

(2) Iida, A., Shinjo, Y., Nukada, H., Yoshikawa, N., Hirahara, S. and Hosoya, M., A Study of the Relationship between Drying State of Toner Image and Transfer Performance in the Image-On-Image (IOI) Color Process Using Liquid Toner, Proceedings of the International Congress of Imaging Science (:ICIS'02) (SPSTJ \& ISJ), (2002), pp.596-597.

( 3 ) Hirahara, S., Watanabe, T., Saito, M., Iida, A., Ishii, K. and Hosoya, M., Liquid Toner Image Transfer Using Shearing Stress, Journal of the Imaging Society of Japan, (in Japanese), Vol.42, No.1 (2003), pp.17-23.

( 4 ) Iida, A., Shinjo, Y., Nukada, H., Yoshikawa, N., Hirahara, S. and Hosoya, M., Relationship between Drying State of Toner Image and Transfer Performance in the IOI Color Process Using Liquid Toner, Journal of the Imaging Society of Japan, (in Japanese), Vol.42, No.1 (2003), pp.24-31.

( 5 ) Hirahara, S., Iida, A., Nukada, H., Shinjo, Y. and Hosoya, M., Liquid Toner Image Transfer Using Shearing

Stress [2], Journal of the Imaging Society of Japan, (in Japanese), Vol.42, No.2 (2003), pp.112-117.

( 6 ) Mochizuki, M. and Kurotori, T., Transition and Development of LLT Copy Machine, Journal of the Society of Electrophotography of Japan, (in Japanese), Vol.26, No.3 (1987), pp.270-276.
( 7 ) Coyne, J.C. and Elrod, H.G., Jr., Conditions of the Rupture of a Lubricating Film. Part 1: Theoretical Model, Transactions of the ASME, Ser. F, Journal of Lubrication Technology, Vol.92, No.3 (1970), pp.451-456.

( 8 ) Savage, M.D., Cavitation in Lubrication. Part 1. On Boundary Conditions and Cavity-Fluid Interfaces, Journal of Fluid Mechanics, Vol.80, Part 4 (1977), pp.743-755.

(9) Reynolds, O., On the Theory of Lubrication and Its Application to Mr. Beauchamp Tower's Experiments, Including an Experimental Determination of the Viscosity of Olive Oil, Philosophical Transactions of the Royal Society of London, A177 (1886), pp.157-234.

(10) Dowson, D. and Higginson, G.R., Elasto-Hydrodynamic Lubrication, (1977), Pergamon Press.

(11) Neumann, A.W., Contact Angles and Their Temperature Dependence: Thermodynamic Status, Measurement, Interpretation and Application, Advances in Colloid and Interface Science, Vol.4 (1974), pp.105-191.

(12) Chen, Y.L., Helm, C.A. and Israelachvili, J.N., Molecular Mechanisms Associated with Adhesion and Contact Angle Hysteresis of Monolayer Surfaces, Journal of Physical Chemistry, Vol.95 (1991), pp.1073610747.

(13) Drelich, J., Wilbur, J.L., Miller, J.D. and Whitesides, G.M., Contact Angles for Liquid Drops at a Model Heterogeneous Surface Consisting of Alternating and Parallel Hydrophobic/Hydrophilic Strips, Langmuir, Vol.12 (1996), pp.1913-1922.

(14) Thompson, P.A., Brinckerhoff, W.B. and Robbins, M.O., Microscopic Studies of Static and Dynamic Contact Angles, Journal of Adhesion Science and Technology, Vol.7, No.6 (1993), pp.535-554.

(15) Hoffman, R., A Study of the Advancing Interface1. Interface Shape in Liquid-Gas Systems, Journal of Colloid and Interface Science, Vol.50, No.2 (1975), pp.228-241.

(16) de Gennes, P.G., Wetting: Statics and Dynamics, Reviews of Modern Physics, Vol.57, No.3, Part 1 (1985) pp.827-86. 